\title{
El aprendizaje autorregulado y los periódicos escolares
}

\section{Self-regulated learning and school newspapers}

DOI: $10.46932 / \mathrm{sfjdv2n2-209}$

Received in: March 1st, 2021

Accepted in: May 30th, 2021

\section{Claudio Marrucci}

Doctorando en Educación en la Universidad Complutense de Madrid. M.A. en Profesorado de literatura castellana. M.A. en Comparative Literature. B.A. en Comparative Literature.

Current Institution: Universidad Complutense de Madrid

Full address: Claudio Marrucci, via Nostra Signora di Lourdes 126, 00167, Roma (Italy)

E-mail: claudiom@ucm.es

\section{RESUMEN}

Los periódicos escolares tienen una larga tradición educativa: hay ejemplos que se remontan al siglo pasado, es decir, cuando la mayoría de las teorías y de los enfoques pedagógicos modernos todavía tenían que desarrollarse. Sin embargo, la literatura científica sobre esta actividad es relativamente escasa. Esta tesis se propone investigar los periódicos escolares desde el punto de vista del aprendizaje autorregulado. En particular, el objetivo de esta investigación es comprobar si hay una relación positiva entre la autorregulación y la participación en proyectos de periódicos escolares. Para alcanzar esta meta, se estudiarán tres grupos de estudiantes que acuden al mismo centro: el IIS Luigi Enaudi, en Roma. De los tres grupos, uno realizará un periódico web, otro un periódico tradicional y el último será un grupo de control. La metodología adoptada será mixta: cualitativa y cuantitativa. El diseño del análisis cualitativo se basará en un estudio de casos a través de entrevistas abiertas a estudiantes y docentes y a través de la observación directa de unas reuniones de redacción. Para el análisis cuantitativo el diseño será cuasi experimental y se prevé el uso de una versión del Motivated Strategies for Learning Questionnaire (MLSQ) validada para estudiantes italianos de secundaria por parte de un equipo de profesores de la Università Cattolica del Sacro Cuore. En cuanto a los resultados, se espera que el grupo del periódico web sea el que mejor logre autorregular su aprendizaje, siendo las características de su proyecto las más cercanas a las premisas teóricas de la autorregulación del aprendizaje.

Palabras clave: aprendizaje autorregulado, autorregulación del aprendizaje, periódico escolar

\begin{abstract}
School newspapers have a long educational tradition: there are examples that go back to the last century, that is, when most theories and modern pedagogical approaches still had to be developed. However, the scientific literature on this activity is relatively scarce. This thesis aims to investigate school newspapers from the point of view of Self-Regulated Learning. In particular, the objective of this research is to check if there is a positive relationship between self-regulation and participation in school newspaper projects. To achieve this goal, three groups of students will be studied who come to the same school: the IIS Luigi Enaudi, in Rome. Of the three groups, one will produce a web newspaper, another a traditional newspaper and the last one will be a control group. The adopted methodology will be mixed: qualitative and quantitative. The design of the qualitative analysis will be based on a case study through open interviews to students and teachers and through the direct observation of writing meetings. For the quantitative analysis the design will be quasi-experimental and it will be used a version of the Motivated Strategies for Learning Questionnaire (MLSQ) validated for Italian high school students by a team of professors of the Università Cattolica del Sacro Cuore. Regarding the results, it is expected that the group of the web
\end{abstract}


newspaper will be the one that best manages to self-regulate their learning, being the characteristics of their project the closest to the theoretical premises of Self-Regulated Learning.

Keywords: SRL, Self-Regulated Learning, School Newspapers

\section{INTRODUCCIÓN}

Hay ejemplos de periódicos escolares que remontan al siglo pasado, sin embargo, son muchos los centros en España y en Europa que siguen proponiendo esta actividad a sus estudiantes.

De hecho, la literatura científica que ha tratado los periódicos escolares siempre ha destacado las excelencias educativas de esta práctica respecto al desarrollo de las destrezas lingüísticas, de las competencias sociales debidas a las dinámicas grupales, de los beneficios en las habilidades manipulativa del niño/a o de las destrezas informáticas del adolescente, etc. Sin embargo, parecen ser relativamente escasas las aproximaciones objetivas basadas en la medición de progresos concretos en estas áreas: los textos existentes se refieren a técnicas de impresión, modelos de utilización de la prensa en la escuela, resumen de experiencias, o propuestas de trabajo con la utilización del periódico escolar en el currículo (Teruel Salmerón, 2015). Esta tesis se propone investigar los periódicos escolares desde el punto de vista del aprendizaje autorregulado comprobando si hay una relación positiva entre la autorregulación y la participación en proyectos de periódicos escolares.

\section{ANTECEDENTES Y PROBLEMA DE INVESTIGACIÓN}

Si bien hay una escasa bibliografía sobre los periódicos escolares y el aprendizaje autorregulado, hay relevantes estudios sobre el aprendizaje autorregulado y la escritura de ensayos o los trabajos de grupos (Monique Boekaerts, Pintrich, \& Zeidner, 2005; Schunk \& Zimmerman, 1998).

El concepto de aprendizaje autorregulado apareció en la escena científica en 1986, durante el congreso anual del American Educational Research Association con la publicación del Contemporary Educational Psychology, en la que se trataron de integrar bajo una sola etiqueta las investigaciones sobre algunos procesos como las estrategias de aprendizaje, la monitorización metacognitiva, la percepción de la autoestima, las estrategias volitivas y el autocontrol. Los autores que participaron en aquel simposio se convirtieron en los grandes clásicos de la literatura científica sobre el aprendizaje autorregulado. Estos son: Monique Boekaerts, Lyn Corno, Steve Graham, Karen Harris, Mary McCaslin, Barbara McCombs, Judit Meece, Richard Newman, Scott Paris, Paul Pintrich, Dale Shunk y Barry Zimmerman.

Uno de los resultados del congreso fue una definición inclusiva del aprendizaje autorregulado, pensado como el grado con el cual los estudiantes son activos desde un punto de vista metacognitivo, motivacional y del comportamiento (Zimmerman, 2008). 
Actualmente el aprendizaje autorregulado sigue siendo un concepto de relevancia que cuenta con el interés científico-académico. De hecho, desde 2013 son más de 2200 los artículos relativos a este constructo publicados y catalogados en la base de datos de la investigación educativa ERIC. Además, desde esa fecha, el Teseo español cuenta con casi una decena de tesis publicadas sobre ese tema.

En el programa del Congreso Europeo de Investigación Educativa ECER 2018, que tuvo lugar en Bolzano, había más de 50 ponencias que mencionaban el aprendizaje autorregulado.

\section{MARCO TEÓRICO}

Los periódicos escolares se podrían definir como un authentic task, o sea, como una tarea compleja, abierta en términos de soluciones, designada para que los estudiantes apliquen sus conocimientos y habilidades en desafíos del mundo real (Gulikers, Bastiaens, \& Kirschner, 2004; Hiebert, 1994; Mims, 1991; Wiggins, 1989, 1990).

En cambio el aprendizaje autorregulado fue definido por Pintrich como:

Un proceso activo y un constructo a través del cual los estudiantes establecen metas para sus aprendizajes y tratan de supervisar, regular y controlar su cognición, motivación y conducta dirigidos y limitados por sus metas y por las características contextuales de su entorno (Pintrich, 2000, p.453).

El elemento clave para definir un aprendizaje "autorregulado" es el empleo y el despliegue de la iniciativa personal, de la perseverancia y de las destrezas adaptativas. El aprendizaje autorregulado es posible solo en contextos en los que existe una cierta posibilidad de elección (Zimmerman, 2001).

En concreto, podemos hablar de aprendizaje autorregulado:

- si los estudiantes pueden elegir o no el participar en una tarea académica. En este sentido se puede hablar de una motivación intrínseca (cuando no hay control por parte de los padres o de los docentes y los objetivos, por ejemplo, son auto-establecidos) o de una motivación auto-motivada (derivada, por ejemplo, de una percepción de autoeficacia)

- si pueden elegir el método de aprendizaje ya sea usando uno planificado (aprendiendo distintas estrategias de aprendizaje como la planificación, la organización o la memorización) o uno automatizado (una vez alcanzado un nivel alto de excelencia académica, las funciones cognitivas llegan a estar automatizadas)

- si pueden elegir los resultados de un desempeño (los estudiantes autorregulados son más autoconscientes de sus resultados y esto les permite más auto-monitorización, control y auto-juicio sobre los resultados de sus desempeños) 
- si pueden controlar el entorno físico (decidiendo dónde y cuándo estudiar o estructurando y modificando el entorno del estudio) y el social (ya sea decidiendo con quién estudiar o de quién alejarse).

El aprendizaje autorregulado no es ni una habilidad mental ni una destreza académica, sino que se refiere a un proceso auto-direccionado a través del cual los estudiantes transforman sus habilidades mentales en destrezas académicas orientadas a un objetivo.

Este enfoque percibe el aprendizaje como una actividad que los estudiantes hacen por sí mismos de una manera proactiva, en lugar de un evento que ocurre de manera reactiva como resultado de una experiencia de enseñanza (Zimmerman, 1989).

Hasta hoy existen sietes grandes teorías con las que se ha analizado el paradigma del aprendizaje autorregulado: la operante, la fenomenológica, la del procesamiento de la información, la socio-cognitiva, la volitiva, la vygotskiana y la constructivista (Zimmerman, 2001). Cada teoría sobre el aprendizaje autorregulado actúa como si fuera una familia de la que surgen diferentes modelos, pero todos con características comunes.

Sin embargo, en los años 2000, Pintrich logró elaborar un modelo general del aprendizaje autorregulado General Framework of Self-Regulation Learning (Garcia \& Pintrich, 1996; Pintrich \& de Groot, 1990; Pintrich, Roeser, \& De Groot, 1994; Pintrich, 2000) que actualmente es el más empleado. Según este modelo, el aprendizaje autorregulado se compone de cuatro fases: planificación, autoobservación, control y reflexión. Para cada una de estas fases, las actividades autorregulatorias se desarrollan en cuatro áreas distintas: la cognitiva, la motivacional y afectiva, la comportamental y la contextual.

- Las actividades autorregulatorias que ocurren en la fase de planificación incluyen: el establecimiento de metas y la activación de conocimientos previos y metacognitivos (área cognitiva); la adopción de metas y de juicios sobre la eficacia, la activación de las creencias sobre el valor de la tarea y la activación del interés personal y los afectos (área afectiva y motivacional); la planificación del esfuerzo y del tiempo (área comportamental) y la percepción de la tarea y del contexto (área contextual)

- De manera similar, la auto-observación consiste en: conciencia y auto-observación de la cognición (área cognitiva); conciencia y auto-observación de la motivación y del afecto (área afectiva y motivacional); conciencia y auto-observación del esfuerzo, del empleo del tiempo y de la necesidad de ayuda (área comportamental) y conciencia y auto-observación de las condiciones de la tarea y del contexto (área contextual) 
- Las actividades de control se refieren a: uso de estrategias cognitivas y meta- cognitivas (área cognitiva); uso de estrategias de control de la motivación y del afecto (área afectiva y motivacional); incremento o disminución del esfuerzo, persistencia y búsqueda de ayuda (área comportamental) y cambios en los requerimientos de la tarea y en las condiciones del contexto (área contextual)

- Por último, la fase de reflexión incluye: juicios cognitivos y atribuciones (área cognitiva); reacciones afectivas (área afectiva y motivacional); elección del comportamiento (área comportamental) y evaluación de la tarea y del contexto (área contextual) (Torrano, Montalvo, \& Torres, 2018).

Un estudiante puede pasar por estas cuatro fases a medida que realiza una tarea. Sin embargo, estas fases representan una secuencia general que no está lineal o jerárquicamente estructurada: estas fases pueden darse de forma simultánea y dinámica, o pueden producir varias interacciones entre los diferentes componentes y procesos (Garcia \& Pintrich, 1994; Pintrich \& de Groot, 1990; Pintrich, 1989).

Se puede pensar en la autorregulación del aprendizaje como en una competencia que se enseña, se aprende y se mide. Los métodos de enseñanza más recurrentes son: la enseñanza directa de estrategias, el modelado, la práctica guiada y autónoma de las estrategias, la auto-observación, el apoyo social y su retiro en el momento en que el estudiante ha alcanzado cierto grado de participación responsable y la autorreflexión (Torrano et al., 2018).

En cuanto a la medición uno de los hallazgos más importantes de Pintrich ha sido la elaboración de The Motivated Strategies for Learning Questionnaire (MSLQ), uno de los auto-informes más utilizados para medir el aprendizaje autorregulado (Pintrich, Smith, García, \& McKeachie, 1991). Se trata de un cuestionario de 81 ítems que refleja el modelo teórico de Pintrich General Framework of Self-Regulation Learning (Garcia \& Pintrich, 1994; Pintrich \& de Groot, 1990; Pintrich, 1989). Su objetivo es medir los diferentes componentes motivacionales y el uso de estrategias de aprendizaje en un curso o en una materia específica. Una de las ventajas de este instrumento es que se ha aplicado y validado en distintos niveles educativos, tanto universitarios como no universitarios. En nuestro caso utilizaremos una versión del (MLSQ) validada para estudiantes italianos de secundaria por parte de un equipo de investigadores de la Università Cattolica del Sacro Cuore (Olivari, Bonanomi, Gatti, \& Confalonieri, 2015).

\section{PREGUNTAS O HIPÓTESIS DE INVESTIGACIÓN}

El objetivo de esta investigación es comprobar si hay una relación positiva entre la autorregulación y la participación en proyectos de periódicos escolares.

Se plantean desde este objetivo dos hipótesis de trabajo: 
H1: Existen diferencias significativas en el nivel de competencia autorregulatoria en función de la participación o no en proyectos previos de periódicos escolares.

$\mathrm{H} 2$ : Existen diferencias significativas en el nivel de competencia autorregulatoria en función del tipo de gestión (auto-gestión y co-gestión) del periódico.

\section{METODOLOGÍA}

La muestra objeto de estudio se basa en tres grupos.

El primer grupo está compuesto por 27 estudiantes de secundaria y está llevando a cabo un proyecto europeo que prevé la redacción de un periódico web. La participación al proyecto es voluntaria, los estudiantes que participan tienen que organizarse de forma autónoma en una redacción, elegir qué noticias tratar y por qué, elegir los tiempos de trabajo (por ejemplo terminando las tareas que se han autoestablecido en casa). Por estas razones se puede hablar de periódico auto-gestionado.

El segundo grupo, está compuesto por 20 estudiantes de secundaria y lleva a cabo un periódico escolar tradicional. Los estudiantes pueden elegir si participar o no así como los tiempos de trabajo. Sin embargo, la toma de decisiones está compartida con el docente. Por esto se puede hablar de periódico cogestionado.

El tercer grupo es un grupo de control de 32 estudiantes de secundaria que nunca ha participado en proyectos de periódicos escolares.

La metodología es mixta: cualitativa y cuantitativa.

El diseño del análisis cualitativo se basa en un estudio de casos llevado a cabo a través de entrevistas abiertas a estudiantes y docentes y a través de la observación directa de unas reuniones de redacciones, en ambos periódicos. Las observaciones directas y las entrevistas serán directa a averiguar cómo ocurre la toma decisión (por ejemplo si hay un grupo de pares que tiene preeminencia sobre otro grupo) cómo se organizan y se dividen las tareas.

Para el análisis cuantitativo el diseño será cuasi experimental y se prevé el uso de una versión adaptada del MLSQ (Olivari, Bonanomi, Gatti, \& Confalonieri, 2015). Se prevé hacer un pre-test y un post-test a los tres grupos objeto de estudio, luego se analizarán los resultados a través un análisis estadístico inferencial.

El instrumento del análisis cuantitativo es una versión del $M L S Q$ reducida a 25 ítems, subdivididos en 5 grupos (auto-eficacia, motivación intrínseca, estrategias de aprendizaje, ansiedad por test, autorregulación). Sigue una escala de tipo Likert de 7 valores (1 muy en desacuerdo, 7 muy de acuerdo). El instrumento está validado para estudiantes italianos de escuela secundaria. Tiene un Alpha de Cronbach de 0,83 (Olivari, Bonanomi, Gatti, \& Confalonieri, 2015) 


\section{RESULTADOS ALCANZADOS HASTA EL MOMENTO}

Hasta el momento se ha avanzado en la profundización del marco teórico que ha llevado a la construcción del instrumento necesario para el análisis cuantitativo. Además se ha tomado contacto con los grupos y en Septiembre 2019 comenzarán las tareas objeto de estudio.

\section{PERSPECTIVA DE CONTINUIDAD DE LA INVESTIGACIÓN}

Como hemos señalado al principio, son relativamente escasos los estudios que miden los progresos educativos relacionados con los periódicos escolares, no obstante, tradicionalmente, la literatura científica siempre ha destacado las excelencias educativas de esta práctica (Teruel Salmerón, 2015). Este estudio es una de las primeras investigaciones que intenta analizar la eficacia educativa de los proyectos de periódicos escolares. Por esto nos hemos centrado en la autorregulación del aprendizaje: un estudiante autorregulado es un estudiante que en su carrera académica "ha aprendido a aprender" y este nos ha parecido el aspecto más relevante a la hora de analizar la eficacia educativa de los periódicos escolares.

De hecho esta tesis intenta comprobar si realmente hay una relación positiva entre el aprendizaje autorregulado y los proyectos de periódicos escolares; o sea, nuestro intento es verificar si los proyectos de periódicos escolares ayudan efectivamente a los estudiantes a hacerse autónomos y conscientes de su propios procesos de aprendizaje, confirmando la valencia educativa que siempre se ha atribuido a los periódicos escolares y que, pero, casi nunca se ha medido de manera objetiva.

En continuidad con estas tesis podrían suponer investigaciones sobre las aportaciones de los proyectos de periódicos escolares en términos de competencia lingüística y digital. 


\section{REFERENCIAS}

Boekaerts, M., Pintrich, P. R., \& Zeidner, M. (2005). Handbook of self-regulation. San Diego: Academic Press.

Garcia, T., \& Pintrich, P. R. (1994). Regulating motivation and cognition in the classroom: The role of self-schemas and self-regulatory strategies. In D. H. Schunk \& B. J. Zimmerman (Eds.), Self-regulation of learning and performance: Issues and educational applications.

Garcia, T., \& Pintrich, P. R. (1996). The effects of autonomy on motivation and performance in the college classroom. Contemporary Educational Psychology, 447-486.

Gulikers, J. T. M., Bastiaens, T. J., \& Kirschner, P. A. (2004). A Five-Dimensional Framework for Authentic Assessment. Educational Technology, Research and Development, 52(3), 67-86.

Hiebert, E. H. (1994). Becoming literate through authentic tasks: Evidence and adaptations. In R. B. Ruddell, M. R. Ruddell, \& H. Singer (Eds.), 4th ed. BT - Theoretical models and processes of reading.

Mims, C. (1991). Authentic Learning: A Practical Introduction \& Guide for Implementation. The Meridian Journal.

Olivari, M., Bonanomi, A., Gatti, E., \& Confalonieri, E. (2015). Psychometric properties of the Motivated Strategies For Learning Questionnaire (MSLQ) among Italian high school students. 14th European Congress of Psychology.

Panarese, P., \& Tumolo, E. (2005). Piccoli inviati speciali. Roma: Carocci.

Pintrich, P.R., \& de Groot, E. V. (1990). Motivational and self-regulated learning components of classroom academic performance. Journal of Educational Psychology, 82(1), 33-40.

Pintrich, P.R., Roeser, R. W., \& De Groot, E. A. M. (1994). Classroom and individual differences in early adolescents' motivation and self-regulated learning. The Journal of Early Adolescence, 14(2), 139-161.

Pintrich, Paul R, Smith, D., García, T., \& McKeachie, W. J. (1991). A Manual for the Use of The Motivated Strategies for Learning Questionnaire (MSLQ). Ann Arbour: University of Michigan, School of Education.

Pintrich, P.R. (1989). The dynamic interplay of student motivation and cognition in the college classroom. In C. Ames \& M. L. Maehr (Eds.), Advances in Motivation and Achievement. Greenwich, CT: JAI Press.

Pintrich, P.R. (2000). The role of goal orientation in self-regulated learning. In M. Boekaerts, P. R. Pintrich, \& M. Zeidner (Eds.), Handbook of Self-regulation. San Diego, CA: Academic Press.

Schunk, D. H., \& Zimmerman, B. J. (1998). Self-regulated learning : from teaching to self-reflective practice. New York: Guilford Press.

Teruel Salmerón, J. A. (2015). Otra prensa: la escolar. Sevilla: Universidad de Sevilla.

Torrano, F., Montalvo, M., \& Torres, C. G. (2018). El aprendizaje autorregulado: presente y futuro de la investigación. Electronic Journal of Research in Educational Psychology, 2(3), 1-34. 
Wiggins, G. (1989). Teaching to the Authentic Test. Educational Leadership, 46(7), 41-47.

Wiggins, G. (1990). The Case for Authentic Assessment. Eric Digest, 1-4.

Zimmerman, B.J. (1989). Dimension of Academic Self-Regulation: a Conceptual Framework for Education. In D.H. Schunk \& B.J. Zimmerman (Eds.), Self-regulation and performance. Hillsdale: Erlbaum.

Zimmerman, B.J. (2001). Self-Regulated Learning and Academic Achievemente: An Overview. In B.J. Zimmerman \& D.H. Schunk (Eds.), Self-regulated learning and academic achievement: Theoretical perspectives (2nd ed.) (2nd ed., pp. 1-37, Chapter x, 322 Pages).

Zimmerman, B.J. (2008). Investigating Self-Regulation and Motivation: Historical Background, Methodological Developments, and Future Prospects. American Educational Research Journal, 45(1), 166-183. 